Revue internationale d'éducation de Sèvres

34 | décembre 2003

La formation professionnelle initiale: une question de société

\title{
États-Unis, Canada : pour ou contre la mixité scolaire?
}

\section{Bernadette Plumelle}

\section{OpenEdition}

\section{Journals}

Édition électronique

URL : https://journals.openedition.org/ries/1568

DOI : $10.4000 /$ ries. 1568

ISSN : 2261-4265

Éditeur

France Education international

Édition imprimée

Date de publication : 1 décembre 2003

Pagination : $20-22$

ISBN : 978-2-8542-0558-9

ISSN : $1254-4590$

Référence électronique

Bernadette Plumelle, «États-Unis, Canada : pour ou contre la mixité scolaire ? », Revue internationale d'éducation de Sèvres [En ligne], 34 I décembre 2003, mis en ligne le 11 avril 2012, consulté le 09 juillet 2021. URL : http://journals.openedition.org/ries/1568 ; DOI : https://doi.org/10.4000/ries.1568

Ce document a été généré automatiquement le 9 juillet 2021.

(c) Tous droits réservés 


\title{
États-Unis, Canada : pour ou contre la mixité scolaire?
}

\author{
Bernadette Plumelle
}

1 L'introduction de la mixité à l'école représente une des transformations majeures du $\mathrm{XX}^{\mathrm{e}}$ siècle en matière d'organisation scolaire. Les États-Unis, la Grande-Bretagne et les pays du nord de l'Europe ont été des pays pionniers dans ce domaine; les pays latins, dont la France, ont adopté ce système plus tardivement mais la mixité, appelée aussi co-éducation aux États-Unis, s'est généralisée dans les années soixante et soixante-dix à l'ensemble des pays occidentaux ${ }^{1}$. Dès les années quatre-vingt-dix, le débat sur la question de la mixité, extrêmement vivant au Canada, aux États-Unis et en GrandeBretagne, s'est étendu à tous les pays industrialisés, comme en témoignent les échos rencontrés en France ces derniers mois par le livre du sociologue français Michel Fize². Cette remise en cause se construit autour de trois grands axes: les mauvais résultats scolaires des garçons par rapport aux filles à tous les niveaux de la scolarité ; des choix de disciplines différents selon les sexes et l'augmentation des violences sexistes et sexuelles dont les filles sont victimes, ceci dans les milieux défavorisés.

2 L'analyse des résultats scolaires selon les sexes est relativement récente et date des années quatre-vingt-dix. Les enquêtes internationales comme celle réalisée en 2000 par l'OCDE (PISA) soulignent la faiblesse des performances du sexe masculin; au Canada, les résultats des garçons sont plus faibles sur l'échelle des compétences ${ }^{3}$. La prise de conscience de ce phénomène s'était amorcée au Québec avec le constat d'un taux élevé d'abandon scolaire plus important chez les garçons ${ }^{4}$. Cela fut aussitôt interprété, comme le montrent les termes utilisés par la presse, comme l'expression d'un «malaise des garçons ». On a fini par évoquer, ces dernières années, la remise en cause de la mixité. La crise de l'identité masculine est souvent invoquée. Les garçons seraient discriminés par un système scolaire devenu un milieu féminisé par son encadrement ainsi que par des rythmes et des activités plus favorables aux filles. Les milieux éducatifs québécois ont pris des mesures en faveur des garçons sous forme de programmes dotés d'importantes ressources matérielles et financières. Un universitaire, Janel Gauthier, directeur de programme en psychologie, préconise ${ }^{5}$ le 
retour aux classes non-mixtes jusqu'à la fin du secondaire. Des écoles primaires et secondaires expérimentent déjà la séparation des sexes dans certaines classes. Dans d'autres, certains cours comme l'histoire, les mathématiques et l'éducation physique sont donnés séparément aux garçons et aux filles.

3 Les États-Unis diffèrent dans le traitement de la mixité. Alors même que les établissements scolaires ont été mixtes beaucoup plus tôt que dans d'autres pays ${ }^{6}$, celleci est remise en cause dans les années quatre-vingt-dix par certains groupes féministes qui considèrent que la mixité n'est pas l'environnement scolaire idéal pour les filles. Ils s'appuient sur le résultat d'enquêtes effectuées à cette époque et qui montrent des différences de niveau entre élèves des deux sexes dans les matières scientifiques, à l'exception de la biologie ${ }^{7}$. Certains chercheurs en éducation considèrent que la mixité peut avoir pour effet de renforcer les stéréotypes sexistes: les enseignants ne sollicitent pas de la même manière filles et garçons; les filles sont prises dans une dynamique relationnelle dominée par les garçons; enfin, des disciplines comme les lettres et les sciences sont perçues très différemment selon le sexe, ceci de manière très précoce. Des recherches anglo-saxonnes montrent que les garçons semblent associer des matières jugées "féminines" comme les lettres à des matières ennuyeuses alors que des disciplines «masculines » comme les mathématiques ou la physique sont jugées difficiles par les filles ${ }^{8}$. Dès 1998, l'État de Californie offre une aide financière aux districts scolaires qui souhaitent pratiquer une éducation séparée selon les sexes. Sur six établissements, seuls trois ont continué cette expérience jusqu'à maintenant. En 1996, une école est créée à Harlem (New York), la Young Women's Leadership Academy, pour les adolescentes de milieux défavorisés. La scolarité dans l'établissement dure six ans et affiche d'excellents résultats. On considère que les jeunes filles de milieu populaire scolarisées dans des établissements non-mixtes, protégées ainsi des violences qu'elles ont à subir de la part des garçons, réussissent beaucoup mieux que les autres prouvant ainsi que l'apprentissage séparé agit sur les résultats. Ces écoles ne font pas l'unanimité au sein du pays et certaines organisations comme l'Union américaine pour les libertés civiques (American Civil Liberties Union) ont porté plainte pour discrimination sexiste. Mais ces prises de position dépassent les clivages politiques ordinaires: l'éducation séparée est défendue par des républicains comme par des démocrates ${ }^{9}$. Un décret voté par le Congrès en février 2001 (No Child Left Behind Act) et signé par le président G.W. Bush, le 8 janvier $2002^{10}$, légifère sur ce point. Dans le cadre de ce décret, les écoles publiques sont autorisées à mettre en place des programmes non-mixtes, le mélange entre élèves des deux sexes n'est plus obligatoire dans les écoles publiques.

La mixité scolaire était perçue comme un gage d'égalité des chances entre filles et garçons. Cette appréciation semble contredite dans les faits par les performances différenciées comme par l'absence de coexistence «pacifique » des deux sexes, plus particulièrement dans les milieux défavorisés. Par ailleurs, si les statistiques confirment les progrès, voire la supériorité croissante des filles dans l'ensemble des cursus, les mécanismes d'orientation continuent à être défavorables aux filles. Celles-ci se retrouvent plus fréquemment dans des filières dites « relationnelles» alors que les garçons se tournent davantage vers les filières des sciences et technologies.

$5 \quad$ Faut-il pour autant remettre en cause la mixité scolaire? Les expériences d'éducation séparée, plus développées dans les pays anglo-saxons que dans les autres pays développés, restent encore très marginales mais préfigurent peut-être une tendance plus générale à la différenciation des modalités d'enseignement selon les sexes. 


\section{NOTES}

1. À l'exception de l'Irlande qui comporte encore un nombre élevé d'écoles non mixtes.

2. Les pièges de la mixité scolaire, Paris, 2003, Presses de la Renaissance.

3. Pour certains sociologues, l'appartenance à une classe sociale est un marqueur social bien plus important que le sexe. Des études soulignent que dans les classes privilégiées, les différences garçons-filles au niveau des résultats sont peu marquées alors que dans les milieux socioéconomiques défavorisés, les filles réussissent mieux que les garçons.

4. En 1998, un rapport montrait que plus de $40 \%$ des garçons quittaient l'enseignement sans diplôme contre $26 \%$ des filles.

5. Article paru dans le quotidien québécois Le Soleil le 2 septembre 2003.

6. " À la fin du XIX ${ }^{\mathrm{e}}$ siècle, la mixité dans le système secondaire public existe à peu près partout ", L'éducation des filles aux États-Unis de la période coloniale à nos jours, Malie Montagutelli, OphrysPloton, 2003, p. 63.

7. Les résultats de l'enquête PISA vont dans le même sens. Pour environ la moitié des pays examinés, les garçons obtiennent de meilleurs résultats que les filles en culture mathématique.

8. Pour une analyse détaillée, on pourra consulter l'article de Marie Duru-Bellat, «Une facette implicite du métier d'élève » (p. 183-202) dans De l'égalité des sexes, 1999, CNDP, (Documents Actes et rapports pour l'éducation).

9. C'est le cas par exemple de Hillary Clinton qui a poursuivi ses études supérieures dans l'une des deux universités, très élitistes, réservées aux femmes, le Smith College et le Wellesley College. 10. On trouvera une présentation de l'ensemble de cette réforme éducative dans le $n^{\circ} 30$ de la Revue internationale d'éducation de Sèvres (juin 2002), p. 10-12.

\section{INDEX}

Index géographique : États-Unis, Canada

Mots-clés : discrimination sexuelle, école mixte, éducation mixte, politique éducative

\section{AUTEUR}

\section{BERNADETTE PLUMELLE}

Responsable du centre de ressources documentaires du CIEP 